\title{
Cerebral Microbleeds: Incidence, Imaging Characteristics, Common and Uncommon Causes
}

\author{
Odysseas Kargiotis*, Apostolos Safouris ${ }^{\star \dagger}$, Georgios Magoufis*, Ermioni Papageorgiou*, Maria Fili*, \\ Klearchos Psychogios*, Eleftherios Stamboulis*, Georgios Tsivgoulis ${ }^{\dagger, \ddagger}$ \\ Stroke Unit, Metropolitan Hospital*, Piraeus; Second Department of Neurology, National \& Kapodistrian University of Athens, School of \\ Medicine, "Attikon" University Hospital ${ }^{\dagger}$, Athens, Greece; Department of Neurology, The University of Tennessee Health Science Center ${ }^{\ddagger}$, \\ Memphis, TN, USA
}

Cerebral microbleeds (CMBs) are considered as a marker of cerebral microangiopathy. CMBs can be detected in various clinical conditions, with the most common being cerebral amyloid angiopathy and chronic hypertension. Uncommon causes of CMBs include cerebral autosomal dominant arteriopathy with subcortical infarcts and leukoencephalopathy, Moyamoya disease, Fabry disease, fat embolism, infective endocarditis, prosthetic heart valves, posterior reversible encephalopathy syndrome, brain radiation, cocaine abuse, thrombotic thrombocytopenic purpura, traumatic brain injury, obstructive sleep apnea, and genetic syndromes related to missense mutations of collagen. In this review, after discussing the causes related to CMBs, we present two cases of patients with a history of aortic dissection that underwent brain magnetic resonance imaging for the investigation of acute onset neurological symptoms. Susceptibility-weighted-imaging (SWI) sequences disclosed multiple, small-sized CMBs located predominantly within hemispheric cortical and subcortical areas, with a pattern of preferential involvement of watershed arterial territories. Although both patients had a history of well-controlled arterial hypertension, the pattern of CMB distribution was distinct to that typically observed in chronic hypertension, which is mainly associated with deep or infratentorial CMBs, as well as with matter hyperintensities and lacunes. The differential diagnosis of diffuse CMBs on brain SWI may also include the history of aortic dissection. The patient's history, in association with the pattern of spatial distribution of the lesions on magnetic resonance imaging-SWI, is usually informative.

\section{J Neurosonol Neuroimag 201\$;10(2):So-94}

Key Words: Hemorrhage, Brain; Cerebral amyloid angiopathy; Aneurysm, Dissecting; Magnetic resonance imaging; Embolism, Fat
Received: September 7, 2018

Revised: October 1, 2018

Accepted: October 1, 2018

Address for correspondence: Georgios Tsivgoulis

Second Department of Neurology, National \& Kapodistrian

University of Athens, Rimini 1, Chaidari, Athens 12462, Greece

Tel: $+30-2105832449$

Fax: $+30-2105832449$

E-mail: tsivgoulisgiorg@ya-

hoo.gr

Odysseas Kargiotis

Stroke Unit, Metropolitan Hospital, Ethnarchou Makariou 9 \& Eleftheriou Venizelou 1 , Piraeus 18547, Greece

Tel: $+30-2104809788$

Fax: $+30-2104814887$

E-mail:kargiody@gmail.com

\section{INTRODUCTION}

Cerebral microbleeds (CMBs) are small-sized (less than $10 \mathrm{~mm}$ in diameter), focal, perivascular, hemosiderin depositions that have emerged as an important marker of brain small vessel disease. These lesions are easily depicted by Gradient echo $\mathrm{T}_{2}$ * (GRE-T2*) magnetic resonance imaging (MRI) and susceptibility weighted imaging (SWI) sequences as round or ovoid hypodense foci within the cerebral parenchyma. ${ }^{1}$ SWI can depict iron depositions and small vessels with greater detail than GRE, thus potentially resulting in CMB's overestimation. ${ }^{2}$ However, a comparison study between GRE and SWI sequences in patients with cerebral amyloid angiopathy (CAA) as well as in healthy non-cerebral amyloid angiopathy controls concluded 
that SWI sequences are more reliable and sensitive in detecting CMBs, because of more accurate differentiation of lesions from background and from pial vessels. ${ }^{3}$

\section{PREVALENCE OF CMBS IN HEALTHY INDIVID- UALS}

CMBs may be seen in $3.1 \%$ to $17 \%$ of healthy population, depending on mean age and origin of the population studied, as well as on the technical characteristics and imaging analysis of the MRI-scanner. ${ }^{4}$ In the population-based Rotterdam Study comprising of 1,062 non-demented individuals with a mean age of 69.6 years, the prevalence of CMBs was even higher and increased further with advancing age. Therefore, for any number of CMBs, the authors found a prevalence of $17.8 \%$ and $38.3 \%$, and for multiple CMBs of $5.4 \%$ and $23.3 \%$ in individuals aged 6o-69 and 8o-97 years old, respectively. The presence of lacunar infarcts and of white matter lesions on brain MRI, as well as patient's cardiovascular risk factors were associated with CMBs of deep or infratentorial location. On the contrary, apolipoprotein E (APOE) 84 carriers displayed more often multiple lobar microhemorrhages. It is noteworthy that lower cholesterol levels were associated with the presence of both lobar and deep or infratentorial microbleeds..$^{5}$ In the Framingham Heart Study, CMBs were present in $8.8 \%$ among 1,965 participants, yet this study population was younger, with a mean age of 66.5 years. Again, CMBs were more common in men and their numbers increased with advancing age. Arterial hypertension was linked to deep and mixed (lobar and deep) CMBs, whereas APOE 84 allele with lobar location. Moreover, statins increased the risk of lobar and mixed location CMBs. ${ }^{6}$ A more recent study reported prevalence of $8 \%$ and $16 \%$ for deep/mixed and lobar CMBs, respectively, with chronic brain ischemic lesions correlating well with the presence of CMBs in deep location.7 The largest population study analyzed 2,602 individuals with a mean age of 74.6 years. CMBs were found in $16.8 \%$ (single $12.0 \%$ and multiple $2.6 \%$ ) and were more common in older and male participants, APOE $\varepsilon 4$ allele carriers, hypertensives, statin and antithrombotic medication users, individuals with lower total cholesterol levels and higher total brain volume, and those with cardiovascular disease and ischemic lesions on brain MRI. ${ }^{8}$ Therefore, CMBs are a common MRI finding, and several clinical, demographic and genetic factors contribute to their development (Table 1).

\section{HISTOPATHOLOGICAL AND IMAGING FIND- INGS}

Hypodense foci on T2-weighted MRI sequences were first associated with hemosiderin deposits in the studies by Tanaka et al. ${ }^{9}$ and Fazekas et al. ${ }^{1}$ in 1999. Both studies used $\mathrm{T}_{2}{ }^{*}$-weighted gradient-echo pulse sequences, whereas the autopsy examination identified these hypodense foci as hemosiderin deposits around arteriosclerotic microvessels. There were occasionally small infarctions surrounding the lesions as well as focal accumulations of hemosiderin-containing macrophages. ${ }^{1,9}$ Micro-dissections, micro-aneurysms, micro-calcifications and arteriolar pseudocalcifications may be falsely perceived as CMBs on brain imaging. ${ }^{10}$ On MRI-SWI imaging, CMBs appear as hypointense small areas and can be differentiated from focal calcifications by the use of phase SWI sequences where the later still preserve hypointense features in contrast with the former that develop a positive (high) signal. ${ }^{11}$ In the manuscript by Azad et al. ${ }^{11}$ the following parameters for axial 3D SWI sequences were applied: repetition time/

TABLE 1. Definition and characteristics of cerebral microbleeds

\begin{tabular}{ll}
\hline Definition & Small-sized (<10 mm in diameter), focal, perivascular, hemosiderin depositions \\
Appearance on MRI GRE-T2* or SWI sequences & Round or ovoid hypodense foci \\
Prevalence in healthy population & 3.1 to $38.3 \%$ \\
Risk factors & Age, arterial hypertension, cardiovascular disease, ischemic lesions on brain \\
& MRI, antithrombotic medication use, APOE $\varepsilon 4$, low cholesterol levels, statins \\
\hline
\end{tabular}

MRI; magnetic resonance imaging, GRE; gradient echo, SWI; susceptibility weighted imaging, APOE; apolipoprotein E. 
echo time 54/40; acquisition time 3:40 minutes; slice thickness $3 \mathrm{~mm}$ and flip angle $15^{\circ}$.

A recent study investigated the correlation between MRI-detected CMBs and histopathologically confirmed microbleeds. A total of 25 autopsy cases had $\mathrm{T}^{*}$ sequences displaying at least one CMB. The sensitivity of MRI-T2* sequences in detecting 'true' CMBs was 51.9\%, and the false negative rate was $48.1 \%$. The low accuracy of MRI in this study may reflect the limited diagnostic value of $\mathrm{T}_{2}{ }^{*}$ sequences in detecting $\mathrm{CMBs}$ compared to SWI. ${ }^{12}$

\section{CMBS AND RISK OF INTRACEREBRAL HEMOR- RHAGE AND ISCHEMIC STROKE}

As a marker of small vessel disease, the burden of CMBs in brain MRI is expected to increase the risk of future acute neuro-vascular events. Similarly, patients with previous ischemic or hemorrhagic stroke are more likely to harbor CMBs. In these settings, individuals with recent ischemic stroke or transient ischemic attack (TIA) and CMBs have a significantly elevated risk of subsequent events, both hemorrhagic and ischemic with odds ratios of 8.52 and 1.55 , respectively. ${ }^{13} \mathrm{~A}$ recent meta-analysis found increased odds for stroke recurrence after ischemic stroke or TIA in the presence of CMBs, with the risk of intracerebral hemorrhage (ICH) being again higher. ${ }^{14}$ The same study analyzed also cohorts without previous stroke and found that CMBs were associated with higher risk of ischemic, and mainly, hemorrhagic stroke (odds ratio 3.59 and 7.46, respectively). In addition, MRI-based CMB detection was an independent predictor of all-cause mortality. ${ }^{14}$

Another meta-analysis of prospective studies on ICH recurrence underscored the importance of CMB burden and distribution. Therefore, patients with CAA-related hemorrhage were in greater recurrence risk compared to those with CAA-unrelated hemorrhage. Moreover, multiple CMBs predicted recurrence in both CAA-related and unrelated ICH cases, however, for the later the association was significant only for $>10$ CMBs. ${ }^{15}$

Patients with ischemic stroke and atrial fibrillation should receive long term anticoagulation. The MRIbased detection of CMBs might assist in defining the risk of hemorrhagic intracranial complications. Indeed, CMBs in anticoagulated patients substantially increase the risk of $\mathrm{ICH}$, especially when multiple (>5 CMBs) ${ }^{16}$ The direct oral anticoagulants (DOACs) have shown to cause significantly less brain hemorrhages than vitamin $\mathrm{K}$ antagonists. ${ }^{17}$ In addition, in patients with anticoagulation-related brain hemorrhage, DOACs might be associated with lower hematoma volumes and less severe neurological deficits. ${ }^{18}$ However, whether DOACs are safer in the presence of CMBs is still unknown. A recent MRI follow-up study of 69 anticoagulated patients with atrial fibrillation showed that DOAC-treated patients, unlike warfarin ones, did not develop new CMBs over 1 year. ${ }^{19}$ Future studies investigating whether DOACs cause less brain hemorrhages than warfarin in individuals with multiple CMBs are warranted.

Another important clinical implication related to CMBs refers to acute ischemic stroke treatment. The most fearful complication of intravenous thrombolysis is hemorrhagic transformation that carries very high mortality rates. ${ }^{20} \mathrm{~A}$ recent meta-analysis comprising of 2,479 patients documented a substantial risk induction of symptomatic hemorrhagic transformation in the presence of CMBs at the pre-treatment MRI. This association was even more powerful for cases with $>10$ microbleeds. ${ }^{21}$ Therefore, in the most recent guidelines from the American Heart Association/American Stroke Association there is a statement referring to multiple CMBs that, when more than 10, render the benefits of intravenous thrombolysis uncertain. ${ }^{22}$

\section{COMMON CAUSES OF CMBS}

Brain microhemorrhages in lobar and subcortical distribution are linked to APOE $\varepsilon 2$ and APOE 84 genotype, beta-amyloid deposition burden on $11 \mathrm{C}-\mathrm{PiB}$ positron emission tomography, and histopathological evidence of CAA. ${ }^{10}$ The Boston criteria define probable or possible CAA when patients aged $>55$ years harbor multiple or single lobar, cortical, or cortico-subcortical hemorrhages on computed tomography/MRI, without evidence of an alternative cause for intracranial hemorrhage. ${ }^{23}$ The diagnosis of CAA is further supported by the presence of cortical superficial siderosis on $\mathrm{T}_{2}$ * or SWI sequences. Increased volume of white matter 
hyperintensities indicating leukoaraiosis is another imaging feature of CAA that is suggestive of a chronic state of cerebral hypoperfusion and ischemia (Fig. 1). ${ }^{24}$ The prevalence of CAA pathology in community based studies is strictly age-related and ranges from $8 \%$ to $49 \%$ depending also to the origin of the population analysed. $^{25}$

The induction of an inflammatory angiocentric response around or within the wall of small and medium sized cortical and leptomeningeal arteries with beta-amyloid depositions results in CAA-related inflammation or angiitis. ${ }^{26}$ The imaging characteristics include cortical and subcortical tumefactive confluent edema without infarcts, and at least one cerebral macro- or microbleed, or cortical superficial siderosis. The most common symptoms are rapidly progressive cognitive decline, focal neurological deficits, seizures, and headaches. The prognosis is generally poor with high rates of morbidity and mortality (up to 60\%). ${ }^{27}$ The patients are treated with corticosteroids and cytostatic agents. In Fig. 2, we present a case of CAA-related inflammation with a marked response to corticosteroids.

Conversely, the presence and burden of CMBs, located predominantly in deep cerebral areas, correlate well with the degree of arterial hypertension and the consequences of hypertension on other end organs, such as the left cardiac ventricule. ${ }^{28}$ In patients suffering from ischemic stroke attributed to hypertension
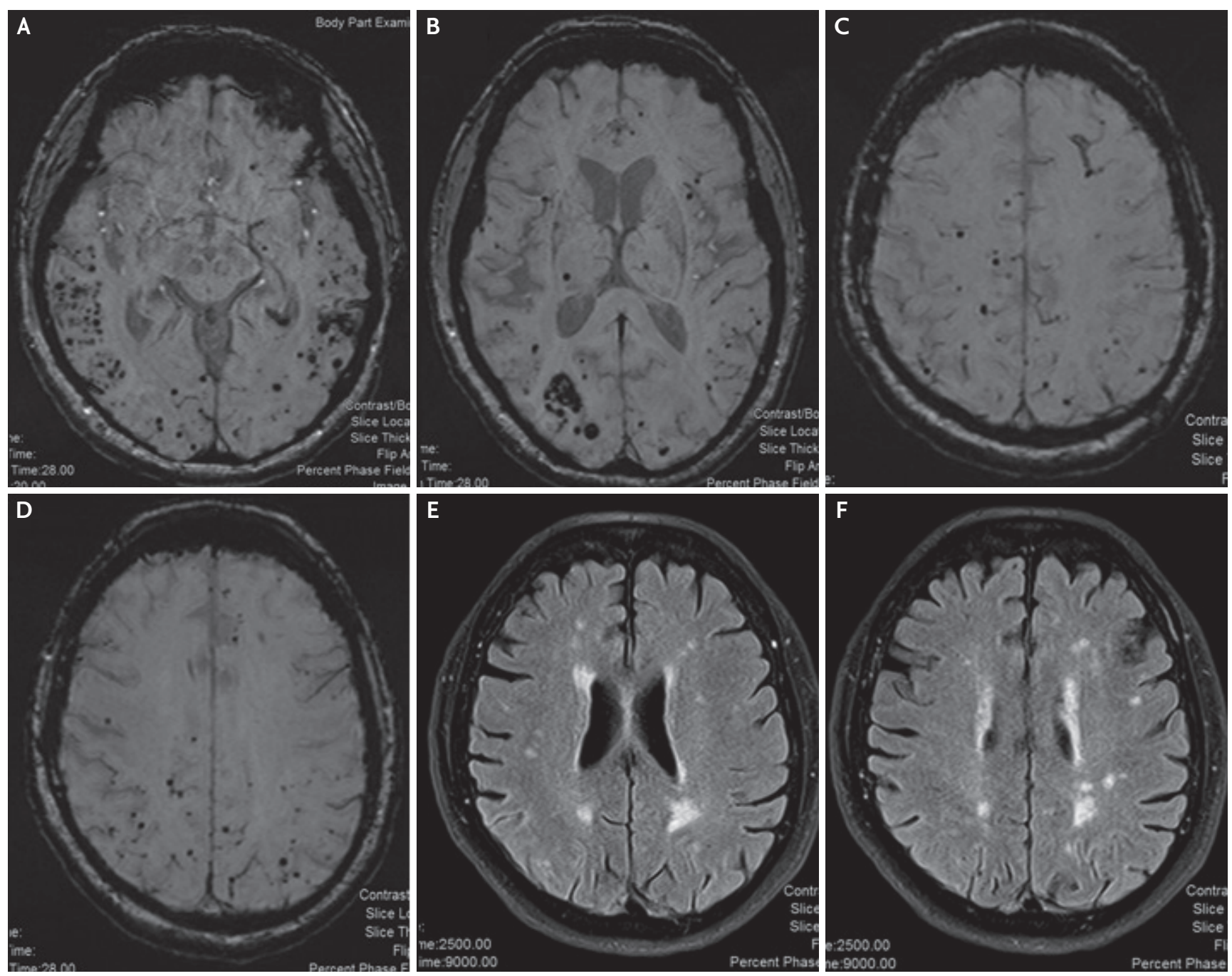

FIG. 1. Magnetic resonance imaging of a 79-year-old female with cerebral amyloid angiopathy. Susceptibility-weighted-imaging sequences showing multiple, predominantly cortical and subcortical cerebral microbleeds (A, B), and superficial siderosis in frontal and parietal sulci (C, D). Fluid-attenuation-inversion-recovery sequences disclosing chronic periventricular, deep white matter and subcortical, ischemic lesions ( $E$, $F$ ). 
related small vessel disease, CMBs may be seen on $\mathrm{T}_{2}$ * sequences in up to $40-60 \%$, and are distributed at the thalamus, putamen, and the subcortical white matter. ${ }^{29}$ Therefore, chronic hypertension causes mostly deep and/or infratentorial CMBs.

Multiple brain microhemorrhages are also observed in individuals with dementia with Lewy bodies, and their numbers correlate well with an increasing white matter lesion burden. Patients suffering from demen- tia with Lewy bodies show similar numbers of CMBs compared with demented subjects diagnosed with Alzheimer's disease, whereas their distribution involves mostly the occipital lobes. ${ }^{30}$

CMBs are commonly encountered after head trauma. The lesions are usually seen in the frontal, temporal and parietal lobes. In cases with a history of more severe head impact, MRI may disclose microhemorrhages in the corpus callosum and deep white matter tracts,
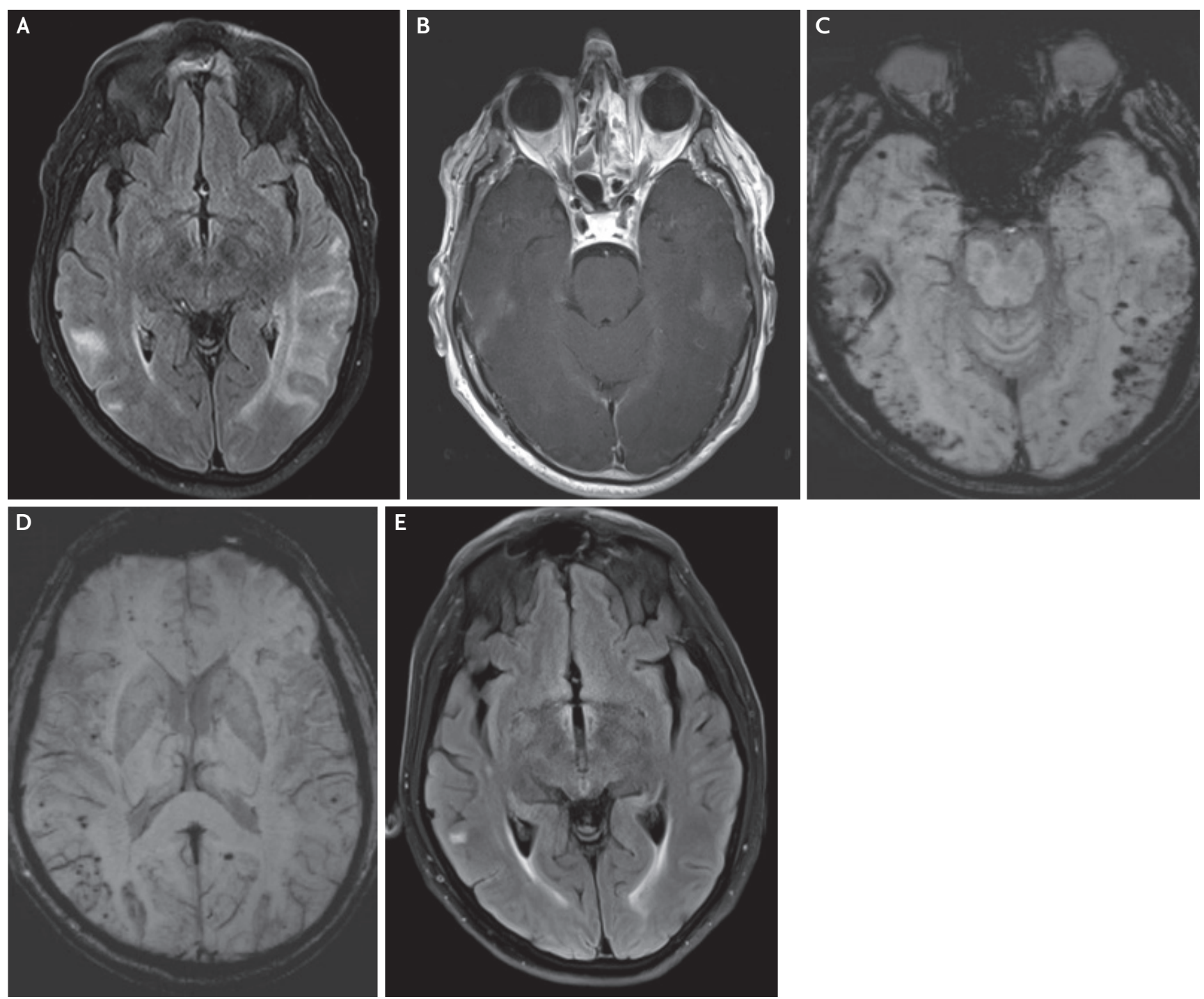

FIG. 2. Cerebral amyloid angiopathy-related inflammation: a 71-year-old male presented with multiple transient episodes of aphasia and difficulty in concentrating. Brain MRI disclosed extensive, bilateral and asymmetric, confluent T2/FLAIR hyperintense areas in the occipital and temporal lobes, with some focal contrast enhancement, consistent with cerebral edema (A, B). Multiple cortical/subcortical microbleeds, predominantly in the posterior areas were also suggestive of an underlying pathology of CAA (C, D). The clinical history and the imaging data fulfilled all diagnostic criteria for probable CAA-related inflammation. The patient received high-dose corticosteroid treatment with significant clinical and imaging amelioration, as shown in the follow-up MRI I month later (E). MRI; magnetic resonance imaging, FLAIR; fluid attenuated inversion recovery; CAA; cerebral amyloid angiopathy. 
and these findings are associated with diffuse axonal injury and subsequent cognitive impairment, predominantly involving executive functions and attention. ${ }^{29,31}$

\section{UNCOMMON CAUSES OF CMBS}

\section{Cerebral autosomal dominant arteriopathy with sub- cortical infarcts and leukoencephalopathy (CADASIL)}

CADASIL is a progressive, non-atherosclerotic, amyloid-negative, hereditary, small vessel disease caused by mutations in the Notch 3 gene. The syndrome was first described 30 years ago in a French family. ${ }^{32}$ Brain MRI discloses: 1) white matter hyperintensities typically involving, among others areas, the external capsule and the anterior temporal poles; 2) lacunar infarcts in the centrum semi-ovale, thalamus, basal ganglia, and pons; and 3) CMBs located predominantly in the thalami (Fig. 3). ${ }^{33}$ The first report for the presence of brain microhemorrhages on $\mathrm{T} 2{ }^{*}$-weighted sequences in patients with CADASIL and their family members found a prevalence of $31 \%$, with the lesions located more often within the thalamus. ${ }^{34}$ A subsequent study reported an ever higher prevalence of CMBs (69\%) located predominantly in cortical-subcortical regions, the white matter, thalamus, and brainstem. Postmortem examination of 7 brains revealed accumulations of hemosiderin-containing macrophages corresponding to the imaging related microhemorrhages..$^{35} \mathrm{~A}$ recent study of 125 patients with CADASIL found a strong correlation of CMBs with age. Hemorrhagic stroke, dementia, urge incontinence, and statin use were also associated with CMB burden after adjustment for age. ${ }^{36}$ Arterial hypertension further increases CMB burden, which, in turn, is associated with an elevated risk of intracerebral hemorrhage and ischemic stroke. Therefore, strict blood pressure control, smoking abstention, and statin avoidance are recommended for individuals with CADASIL. However, the pathophysiological mechanisms related to CMBs in these individuals may be at least partially distinct from those mechanisms associated to leukoaraiosis and lacunar infarcts. Indeed, a recent study found no significant correlation between CMB and other imaging features of cerebral small vessel disease. On the contrary, the authors documented the well-known association of CMBs with age. ${ }^{37}$ Thus, an effective prevention strategy for the deceleration of CMB development in CADASIL patients is currently unavailable.

\section{Fabry disease}

Fabry disease is an X-linked lysosomal storage disorder caused by alpha-galactosidase A gene mutation resulting in deficient activity of alpha-galactosidase A and progressive lysosomal deposition of globotriaosylceramide in cells throughout the body. The disease may affect the peripheral nerves, skin, kidneys, brain and heart. ${ }^{38}$ Brain MRI shows evidence of small vessel disease, including white matter hyperintensities and CMBs, in almost half of the patients. ${ }^{39}$ CMBs are found in $30 \%$ of cases, more frequently in males, individuals with white matter hyperintensities, and chronic kidney disease. ${ }^{40}$

\section{Type IV collagen alpha 1 (COL4A1) mutations}

Mutations in the gene coding for COL4A1 were initially linked to porencephaly in humans. Later, phenotypic observations in a French family harboring COL4A1 mutations lead to a connection with brain small vessel disease. Diffuse leukoencephalopathy, dilated perivascular spaces, lacunes, and CMBs represent typical brain MRI findings. ${ }^{41}$ Sporadic cases with spontaneous ICH have been found to harbor COL4Aı mutations that lead to defective type IV collagen deposition within the basement membrane of the vessel wall, thus to the development of fragile cerebral vessels that are prone to rupture after minimal trauma, intense activity or anticoagulation treatment..$^{42}$ A study of 52 carriers of COL4A1 mutations reported increased stroke rates, both ischemic and hemorrhagic, with a mean age at presentation of 36 years. Hemorrhages were subcortical and ischemic strokes lacunar, indicating small vessel pathology. The location of CMBs was subcortical. ${ }^{43}$

\section{Posterior reversible encephalopathy syndrome (PRES)}

PRES is characterized by cerebral vasogenic edema and clinical manifestations including headache, seizures, visual disturbances and confusion. PRES might 

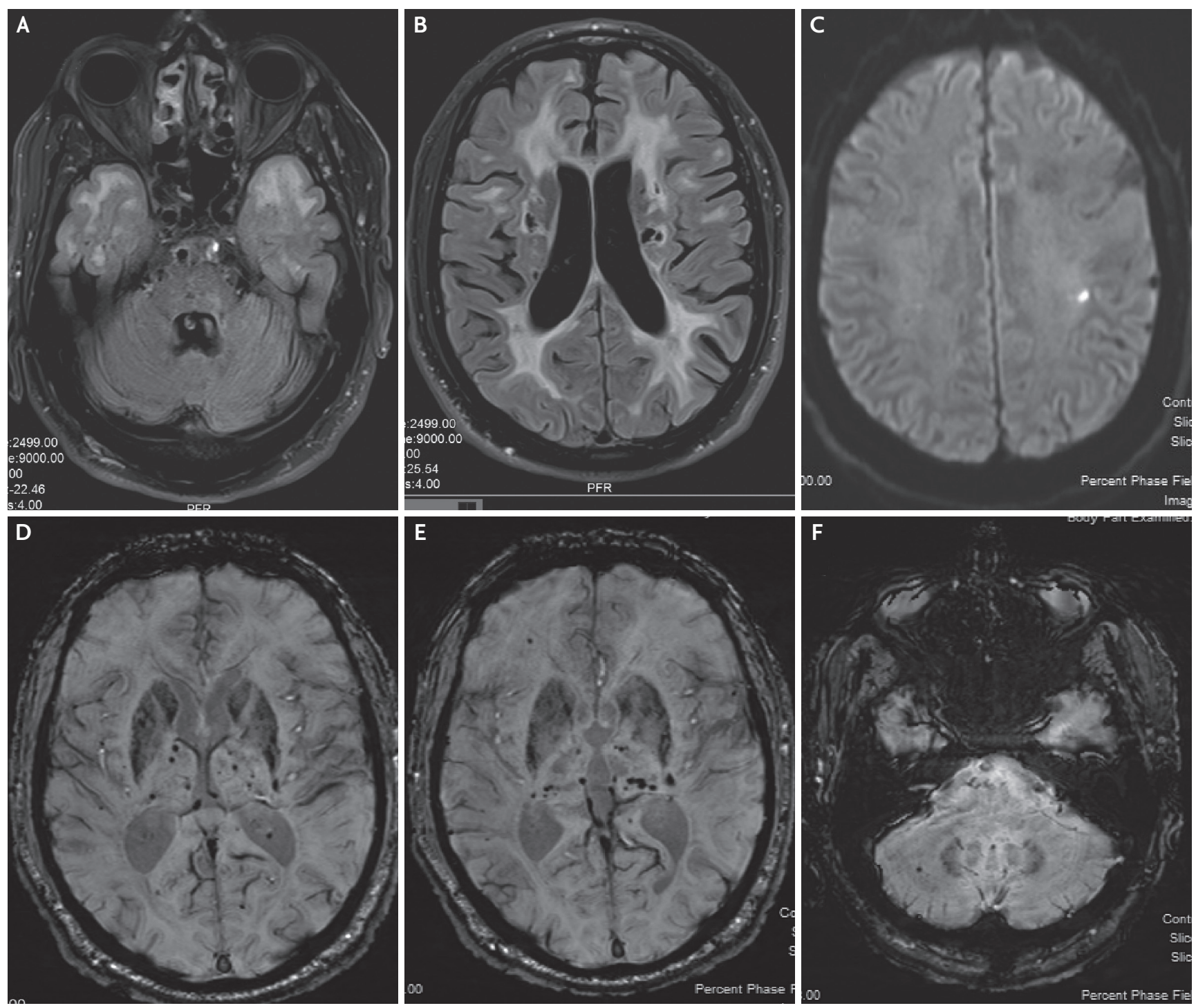

FIG. 3. Brain magnetic resonance imaging of a 58 -year-old patient with cerebral autosomal dominant arteriopathy with subcortical infarcts and leukoencephalopathy. Fluid-attenuated inversion recovery sequence showing white matter lesions involving the anterior temporal poles (A), and the subcortical/deep white matter, as well as bilateral lacunar infarcts (B). An acute lacunar infarct in the left center semiovale (C). Abundant cerebral microbleeds in various regions including the thalamus, cortical and subcortical structures, the cerebellum, and the basal ganglia (D-F).

be idiopathic or secondary to a variety of causes, with the most common being arterial hypertension/hypertasic crisis, transplantation-related immunosuppressive medication, chemotherapy, renal disease and preeclampsia. ${ }^{44} \mathrm{CMBs}$ have been reported in the context of Tacrolimus and thrombotic thrombocytopenic purpura-related PRES. ${ }^{45,46}$ Intracranial hemorrhage might complicate PRES in 15.2-17.3\% of cases. An MRI study of 31 patients disclosed CMBs in $65 \%$ of them. ${ }^{47}$

\section{Infective endocarditis}

Infective endocarditis is a potentially lethal clinical condition, which may provoke systemic embolization in $22 \%$ to $50 \%$ of cases, with up to $65 \%$ of embolic events involving the central nervous system..$^{4} \mathrm{CMBs}$, both in cortical and subcortical locations, are common, detected in up to $57 \%$ of cases, and their presence is a predictor of an impending intracranial hemorrhage. 49,50 Cerebral microhemorrhages on brain MRI are described even in the majority of neurologically asymp- 
tomatic individuals with infective endocarditis. ${ }^{51}$ Another study found that prosthetic heart valve-associated infective endocarditis is strongly related to the presence of CMBs. $^{52}$

\section{Moyamoya disease}

Moyamoya disease is a chronic, steno-occlusive cerebrovascular disease of unknown etiology. The typical imaging features include steno-occlussive lesions of the terminal intracranial internal carotid arteries, with a subsequent development of an abnormal vascular network at the base of the brain. The disease is more commonly encountered in Eastern Asian. There are two different age groups that show pick-incidence of disease presentation, the first between 10-20 and the second between 35-50 years of age. Recurrent ischemic strokes due to hypoperfusion, and intracranial hemorrhages, often intraventricular, may develop over the course of the disease. ${ }^{53} \mathrm{CMBs}$, located in deep and periventricular regions, are seen in $28-46 \%$ of cases although a recent report concerning a European moyamoya patient cohort found a lower prevalence of $12.9 \% . .^{54-56}$ Their presence is associated with dilated anterior choroidal or posterior communicating arteries. Periventricular CMBs increase the risk of intraventricular hemorrhage.

\section{Irradiation}

Brain irradiation is widely used as primary or adjunctive therapy of central nervous system tumors. However, several early or late complications might occur, such as tissue necrosis, white matter injury, brain atrophy, mineralization, microangiopathy, telangiectasia, optic neuropathy, and large vessel vasculopathy. ${ }^{57}$ Radiation-induced vascular injury often involves the small arteries and capillaries leading to endothelial injury, vascular ectasia and microbleeds. An MRI study of 34 patients detected CMBs in 16 (47\%). The time latency between radiation exposure and imaging was 3 months to 9 years, whereas a significant correlation between irradiation dose and CMB prevalence was noticed. ${ }^{5}$ Recently, a latency of 8-22 months between irradiation for glioma and initial appearance of microbleeds has been reported, whereas 2 years after exposure the numbers of CMBs substantially increased. Noticeably, patients that had received antiangiogenic agents had significantly fewer microbleeds. ${ }^{59}$ The clinical significance of irradiation-induced CMBs has been addressed in several studies of various cancer types, including pediatric tumors, that have documented an association of CMBs with cognitive decline. ${ }^{60-62}$

\section{Obstructive sleep apnea}

Obstructive sleep apnea has a high prevalence in general population, whereas it is considered as an important risk factor of cardiovascular disease and mortality. ${ }^{63}$ Recently, moderate to severe obstructive sleep apnea has been linked to CMBs, since higher apnea-hypopnea index was an independent predictor of CMBs after adjustment for age, hypertension, diabetes, and cardiovascular disease. ${ }^{63,64}$ Whether individuals with obstructive sleep apnea harboring CMBs are in greater risk of hemorrhagic or ischemic stroke remains to be elucidated.

\section{Cocaine use}

Cocaine abuse is a well-known cause of stroke, both ischemic and hemorrhagic. Cocaine intake can result in acute arterial hypertensive peaks, as well as may induce cerebral vasoconstriction and vasculitis, with bloodbrain barrier disruption and neuroinflammation. ${ }^{65}$ Particularly small cerebral vessels might become leaky and fragile. Shoamanesh et al. ${ }^{66}$ evaluated the MRI scans from 104 young patients ( $\leq 49$ years) with a history of stroke. CMBs were detected in $17 \%$, and among other risk factors examined, subjects with positive serum toxicology for cocaine were more likely to develop new microbleeds on repeat MRI (67\% vs. $13 \%){ }^{66}$

\section{Hematological disorders}

Thrombotic thrombocytopenic purpura (TTP) and disseminated intravascular coagulation (DIC) are rare hematological disorders with frequent neurological manifestations, such as seizures, ischemic and hemorrhagic stroke. TTP affects the brain through a multisystemic thrombotic microangiopathy and microthrobi formation. Cortical-subcortical as well as deep gray/ white matter CMBs have been described on MRI-GRE 
or SWI sequences of TTP patients, with or without an underlying PRES. ${ }^{46,67,68}$ Similarly, multiple CMBs have been also reported in patients with DIC. ${ }^{69-71}$ In both clinical conditions, it is postulated that intravascular microthrombi and parenchymal hemosiderin deposits surrounding small vessels are accounted for the formation of microbleeds.

\section{Aortic dissection and cerebral fat embolism syn- drome}

1) Cases presentation

A 40-year-old man, with a 3-year history of exten- sive aortic dissection (type I according to the DeBakey

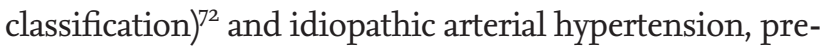
sented with an episode of transient numbness over his left arm. A brain MRI excluded acute ischemic or hemorrhagic lesion. However, SWI sequences disclosed multiple $(\mathrm{n}=2 \mathrm{O})$ CMBs located predominantly in hemispheric cortical/subcortical, as well as cerebellar grey and white matter areas, with a pattern of preferential involvement of watershed arterial territories (Fig. 4AD). T2-sequences were unremarkable except from one lacunar lesion in the left centrum-semiovale and no further evidence of hypertensive small vessel disease (Fig. 4E, F).
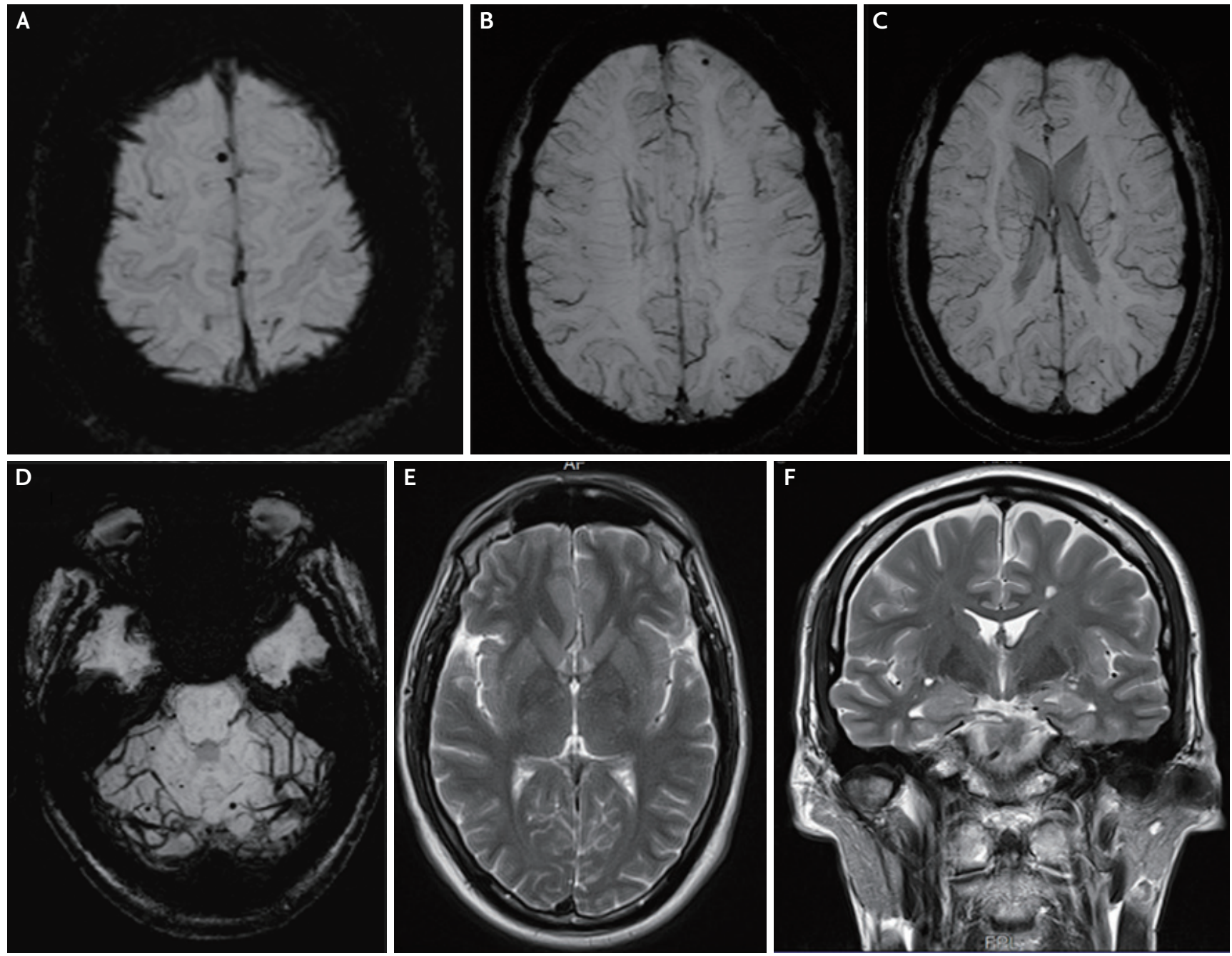

FIG. 4. Brain magnetic resonance imaging of the first case with extensive aortic dissection: brain MRI-SWI showing multiple cerebral microhemorrhages, the majority with small diameter, in hemispheric cortical/subcortical, cerebellar grey and white matter areas and watershed zones, a pattern distinct from that observed in arterial hypertension and cerebral amyloid angiopathy (A-D). T2-sequence verifying the absence of white matter ischemic changes (E). A lacunar infarction is displayed in the left centrum semiovale (F). MRI; magnetic resonance imaging, SWl; susceptibility-weighted-imaging. 
The second case refers to a 65-year-old male with a 10-year history of extensive aortic dissection DeBakey type I, as well as idiopathic arterial hypertension. He underwent urgent brain-MRI because of acute onset left-sided hemiparesis attributed to ischemic stroke, caused by acute thrombosis of the pseudolumen of the dissected aorta and subsequent extension of the chronic dissection with subocclusion of the innominate artery. SWI sequences disclosed multiple $(\mathrm{n}=10)$ CMBs located predominantly in hemispheric cortical and subcortical areas with a pattern of preferential involvement of watershed arterial territories (Fig. 5 B-F). Diffusion-weighted sequences disclosed an acute, right frontal-parietal-temporal cortical ischemic stroke, with no further evidence of hypertensive small vessel disease (Fig. 5A). In both patients, extensive work-up found no damage in other end organs (retina, kidneys, and heart) from chronic hypertension. Moreover, the family history and clinical examination were not suggestive of any hereditary connective tissue disease.

\section{Discussion}

Although the majority of patients with a history of aortic dissection are also hypertensives, CMBs in our patients with aortic dissection displayed a different pattern of spatial distribution compared to that seen in patients with severe arterial hypertension, with more
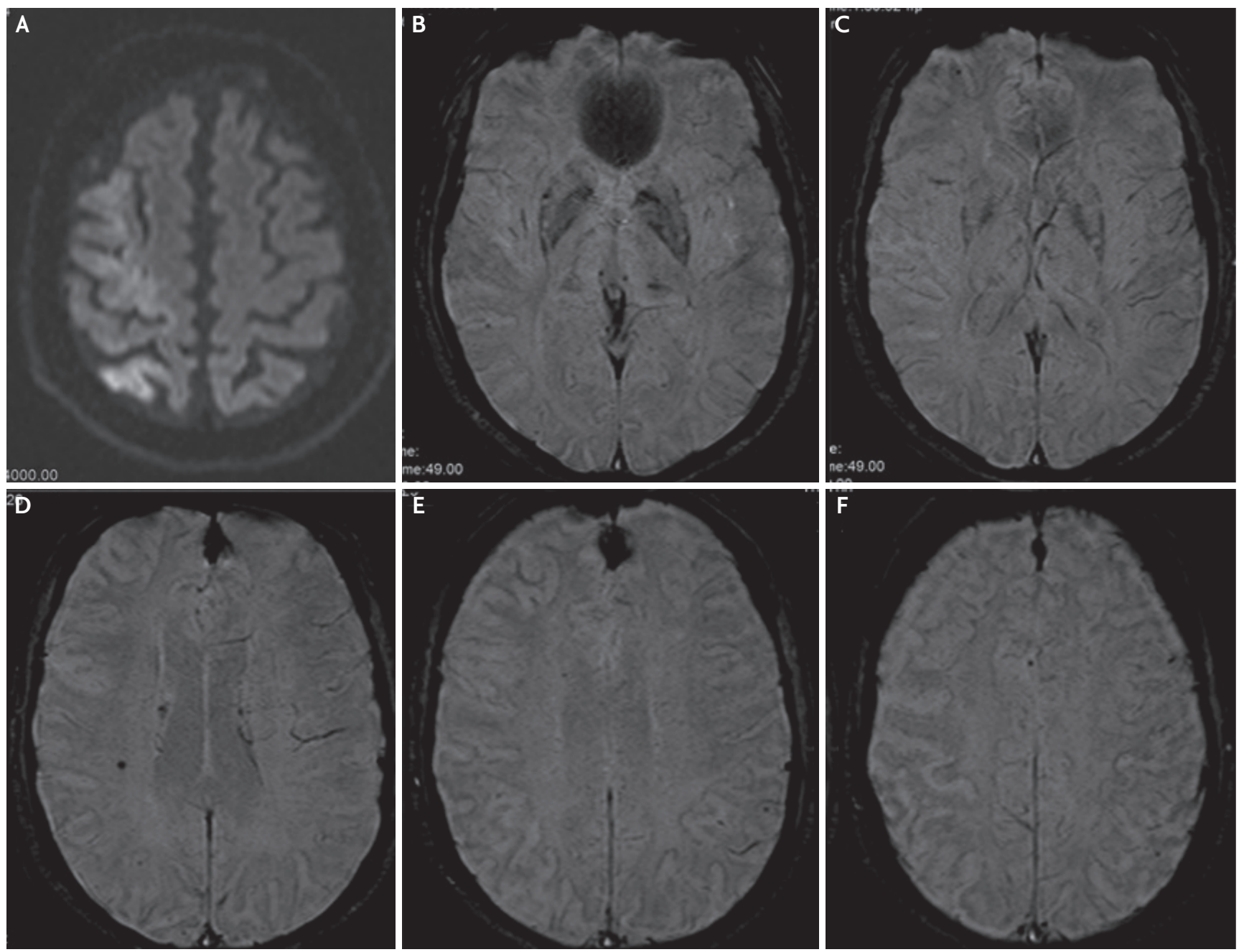

FIG. 5. Brain magnetic resonance imaging of the second case with extensive aortic dissection: diffusion-weighted imaging showing an acute right hemispheric cortical ischemic stroke (A). Brain MRI-SWI showing multiple cerebral microhemorrhages, the majority with small diameter, in hemispheric cortical and subcortical areas, with a distribution mostly within arterial watershed zones (B-F). MRI; magnetic resonance imaging, SWI; susceptibility-weighted-imaging. 
frequent involvement of frontal and occipital lobes and less of deep grey and white matter tracts, a pattern suggestive of preferential localization of CMBs in watershed subcortical arterial zones. A distinct mechanism seems to be responsible for the CMBs development in individuals with aortic dissection. Indeed, CMBs might be a result of fat embolism, a shower of cholesterol microemboli produced from the injured intimal layer of the aorta during acute dissection, subsequently becoming embedded into the cerebral microvasculature. Interestingly, distal cholesterol crystal embolism has been described following aortic dissection. ${ }^{73,74}$

The proposed mechanism of CMB development after aortic dissection is similar to that of the fat embolism syndrome, which is typically precipitated by major trauma with fracture of long bones leading to fat from the bone marrow released into the blood stream. In such cases, MRI-SWI disclose multiple, small sized ( $<3 \mathrm{~mm}$ ) CMBs in watershed territories located in the hemispheric white matter and in the gray and white matter of the cerebellum. ${ }^{29}$ The incidence of cerebral fat embolism in patients with long bone fracture is low, between $0.9 \%$ and $2.2 \% .{ }^{75}$ An autopsy study of a patient who sustained multiple fractures in an automobile accident and subsequently developed fat embolism syndrome revealed abundant CMBs, up to $2-3 \mathrm{~mm}$ in diameter, diffusely distributed in the cerebral white matter (centrum semiovale, deep white matter, internal capsule), but largely sparing the cortex and deep nuclei. However, in the cerebellum the CMBs involved both the white and gray matter. Few hemorrhages were also detected in the brain stem and spinal cord. Yet, the detection of white matter petechiae does not provide a definitive diagnosis of cerebral fat embolism, since an autopsy examination with light microscopy is required. These microhemorrhages occur when fat globules occlude the microvasculature leading to necrosis and hemorrhage in the surrounding brain parenchyma. ${ }^{76}$ Thus, it is suggested that $\mathrm{T}_{2}{ }^{*}$-weighted gradient-echo MRI sequences may serve as a powerful diagnostic tool for the early confirmation of cerebral fat embolism. ${ }^{77}$ Interestingly, cerebral fat embolism, appearing as multiple CMBs on $\mathrm{T}_{2}$ * GRE sequences, has been reported in patients with $\beta$-Thalassemia as a consequence of bone marrow infarcts and necrosis. ${ }^{78}$

Larger case series are needed to confirm the possible association between CMBs and aortic dissection. The broad differential diagnosis of diffuse CMBs on brain MRI may also include the history of aortic dissection. In such cases, the patient's history in association with the pattern of spatial distribution of the lesions on MRI-SWI may guide the final diagnosis.

\section{CONCLUSION}

CMBs are relatively common findings disclosed by $\mathrm{T}_{2}{ }^{*} \mathrm{GRE}$ and SWI MRI sequences. Table 2 presents the causes of MRI-detected CMBs. In the majority of cases they represent imaging markers of small vessel disease

TABLE 2. Causes of cerebral microbleeds

\begin{tabular}{|c|c|c|c|c|}
\hline $\begin{array}{l}\text { Sporadic cerebral small vessel } \\
\text { diseases }\end{array}$ & $\begin{array}{l}\text { Inherited or genetic small } \\
\text { vessel diseases }\end{array}$ & $\begin{array}{l}\text { Hematological } \\
\text { disorders }\end{array}$ & Cardiac disorders & Miscellaneous conditions \\
\hline $\begin{array}{l}\text { Sporadic cerebral amyloid an- } \\
\text { giopathy (including patients } \\
\text { with Alzheimer's disease) } \\
\text { Hypertensive arteriopathy }\end{array}$ & $\begin{array}{l}\text { CADASIL/CARASIL } \\
\text { Fabry's disease } \\
\text { Small vessel diseases caused } \\
\text { by COL4Aı mutations } \\
\text { Hereditary cerebral amyloid } \\
\text { angiopathy }\end{array}$ & $\begin{array}{l}\text { Sickle cell disease } \\
\text { DIC } \\
\text { Thrombotic } \\
\text { thrombocyto- } \\
\text { penic purpura }\end{array}$ & $\begin{array}{l}\text { Cardiac myxoma } \\
\text { Infective } \\
\text { endocarditis }\end{array}$ & $\begin{array}{l}\text { Post-radiation treatment } \\
\text { Traumatic brain injury } \\
\text { PRES } \\
\text { Moyamoya disease } \\
\text { Intravascular lymphomatosis } \\
\text { Obstructive sleep apnea } \\
\text { Cocaine abuse } \\
\text { Aortic dissection } \\
\text { Fat embolism syndrome } \\
\text { Parry-Romberg syndrome }\end{array}$ \\
\hline
\end{tabular}

CADASIL; cerebral autosomal dominant arteriopathy with subcortical ischemic strokes and leukoencephalopathy, CARASIL; cerebral autosomal recessive arteriopathy with subcortical ischemic strokes and leukoencephalopathy, COL4Aı; type IV collagen alpha 1, DIC; disseminated intravascular coagulation, PRES; posterior reversible encephalopathy syndrome. 
and are indicative of an increased risk of future cerebrovascular events and cognitive decline. Their presence and burden might influence treatment strategies, such as the choice of anticoagulation and the management of acute ischemic stroke. ${ }^{79}$ In selected cases, less common causes of CMBs should be considered. ${ }^{80}$ The size and location of microbleeds, as well as the clinical history are helpful for the differential diagnosis. ${ }^{79,80}$ Moreover, the two cases described herein highlight a possible association between history of extensive aortic dissection and detection of MRI lesions indicative of CMBs, which exhibit distinct imaging features and may be a consequence of fat embolism.

\section{Conflicts of Interest}

No potential conflicts of interest relevant to this article was reported.

\section{REFERENCES}

1. Fazekas F, Kleinert R, Roob G, Kleinert G, Kapeller P, Schmidt R, et al. Histopathologic analysis of foci of signal loss on gradient-echo $\mathrm{T} 2{ }^{*}$-weighted MR images in patients with spontaneous intracerebral hemorrhage: evidence of microangiopathy-related microbleeds. AJNR Am J Neuroradiol. 1999;20:637-642.

2. Mittal S, Wu Z, Neelavalli J, Haacke EM. Susceptibility-weighted imaging: technical aspects and clinical applications, part 2. AJNR Am J Neuroradiol. 2009;30:232-252.

3. Cheng AL, Batool S, McCreary CR, Lauzon ML, Frayne $\mathrm{R}$, Goyal M, et al. Susceptibility-weighted imaging is more reliable than $\mathrm{T}_{2}{ }^{*}$-weighted gradient-recalled echo MRI for detecting microbleeds. Stroke. 2013;44:2782-2786.

4. Tsushima Y, Tanizaki Y, Aoki J, Endo K. MR detection of microhemorrhages in neurologically healthy adults. Neuroradiology. 2002;44:31-36.

5. Vernooij MW, van der Lugt A, Ikram MA, Wielopolski PA, Niessen WJ, Hofman A, et al. Prevalence and risk factors of cerebral microbleeds: the Rotterdam scan study. Neurology. 2008;70:1208-1214.

6. Romero JR, Preis SR, Beiser A, DeCarli C, Viswanathan A, Martinez-Ramirez S, et al. Risk factors, stroke prevention treatments, and prevalence of cerebral microbleeds in the Framingham heart study. Stroke. 2014;45:1492-1494.

7. Graff-Radford J, Simino J, Kantarci K, Mosley TH Jr, Gris- wold ME, Windham BG, et al. Neuroimaging correlates of cerebral microbleeds: the ARIC study (Atherosclerosis Risk in Communities). Stroke. 2017;48:2964-2972.

8. Ding J, Sigurðsson S, Jónsson PV, Eiriksdottir G, Meirelles $\mathrm{O}$, Kjartansson O, et al. Space and location of cerebral microbleeds, cognitive decline, and dementia in the community. Neurology. 2017;88:2089-2097.

9. Tanaka A, Ueno Y, Nakayama Y, Takano K, Takebayashi S. Small chronic hemorrhages and ischemic lesions in association with spontaneous intracerebral hematomas. Stroke. 1999;30:1637-1642.

10. Yates PA, Villemagne VL, Ellis KA, Desmond PM, Masters CL, Rowe CC. Cerebral microbleeds: a review of clinical, genetic, and neuroimaging associations. Front Neurol. 2014;4:205.

11. Azad R, Mittal P, Malhotra A, Gangrade S. Detection and differentiation of focal intracranial calcifications and chronic microbleeds using MRI. J Clin Diagn Res. 2017;11:TC19$\mathrm{TC} 23$.

12. Haller S, Montandon ML, Lazeyras F, Scheffler M, Meckel S, Herrmann FR, et al. Radiologic-histopathologic correlation of cerebral microbleeds using pre-mortem and post-mortem MRI. PLoS One. 2016;11:e0167743.

13. Charidimou A, Kakar P, Fox Z, Werring DJ. Cerebral microbleeds and recurrent stroke risk: systematic review and meta-analysis of prospective ischemic stroke and transient ischemic attack cohorts. Stroke. 2013;44:995-1001.

14. Charidimou A, Shams S, Romero JR, Ding J, Veltkamp $\mathrm{R}$, Horstmann $\mathrm{S}$, et al. Clinical significance of cerebral microbleeds on MRI: a comprehensive meta-analysis of risk of intracerebral hemorrhage, ischemic stroke, mortality, and dementia in cohort studies (v1). Int J Stroke. 2018;13:454468.

15. Charidimou A, Imaizumi T, Moulin S, Biffi A, Samarasekera $\mathrm{N}$, Yakushiji Y, et al. Brain hemorrhage recurrence, small vessel disease type, and cerebral microbleeds: a meta-analysis. Neurology. 2017;89:820-829.

16. Charidimou A, Karayiannis C, Song TJ, Orken DN, Thijs $\mathrm{V}$, Lemmens R, et al. Brain microbleeds, anticoagulation, and hemorrhage risk: meta-analysis in stroke patients with AF. Neurology. 2017;89:2317-2326.

17. Katsanos AH, Mavridis D, Parissis J, Deftereos S, Frogoudaki A, Vrettou AR, et al. Novel oral anticoagulants for the secondary prevention of cerebral ischemia: a network meta-analysis. Ther Adv Neurol Disord. 2016;9:359-368.

18. Tsivgoulis G, Lioutas VA, Varelas P, Katsanos AH, Goyal $\mathrm{N}$, Mikulik R, et al. Direct oral anticoagulant- vs vitamin $\mathrm{K}$ 
antagonist-related nontraumatic intracerebral hemorrhage. Neurology. 2017;89:1142-1151.

19. Saito T, Kawamura Y, Sato N, Kano K, Takahashi K, Asanome A, et al. Non-vitamin $\mathrm{K}$ antagonist oral anticoagulants do not increase cerebral microbleeds. J Stroke Cerebrovasc Dis. 2015;24:1373-1377.

20. Tsivgoulis G, Kargiotis O, Alexandrov AV. Intravenous thrombolysis for acute ischemic stroke: a bridge between two centuries. Expert Rev Neurother. 2017;17:819-837.

21. Tsivgoulis G, Zand R, Katsanos AH, Turc G, Nolte CH, Jung $S$, et al. Risk of symptomatic intracerebral hemorrhage after intravenous thrombolysis in patients with acute ischemic stroke and high cerebral microbleed burden: a meta-analysis. JAMA Neurol. 2016;73:675-683.

22. Powers WJ, Rabinstein AA, Ackerson T, Adeoye OM, Bambakidis NC, Becker K, et al. Guidelines for the early management of patients with acute ischemic stroke: a guideline for healthcare professionals from the American Heart Association/American Stroke Association. Stroke. 2018;49:e46-e110.

23. Knudsen KA, Rosand J, Karluk D, Greenberg SM. Clinical diagnosis of cerebral amyloid angiopathy: validation of the Boston criteria. Neurology. 2001;56:537-539.

24. Gregg NM, Kim AE, Gurol ME, Lopez OL, Aizenstein HJ, Price JC, et al. Incidental cerebral microbleeds and cerebral blood flow in elderly individuals. JAMA Neurol. 2015;72:10211028.

25. Chen YW, Lee MJ, Smith EE. Cerebral amyloid angiopathy in East and West. Int J Stroke. 2010;5:403-411.

26. Miller-Thomas MM, Sipe AL, Benzinger TL, McConathy J, Connolly S, Schwetye KE. Multimodality review of amyloid-related diseases of the central nervous system. Radiographics. 2016;36:1147-1163.

27. Auriel E, Charidimou A, Gurol ME, Ni J, Van Etten ES, Martinez-Ramirez $\mathrm{S}$, et al. Validation of clinicoradiological criteria for the diagnosis of cerebral amyloid angiopathy-related inflammation. JAMA Neurol. 2016;73:197-202.

28. Lee SH, Park JM, Kwon SJ, Kim H, Kim YH, Roh JK, et al. Left ventricular hypertrophy is associated with cerebral microbleeds in hypertensive patients. Neurology. 2004;63:16-21.

29. Schrag M, Greer DM. Clinical associations of cerebral microbleeds on magnetic resonance neuroimaging. J Stroke Cerebrovasc Dis. 2014;23:2489-2497.

30. Gungor I, Sarro L, Graff-Radford L, Zuk SM, Tosakulwong N, Przybelski SA, et al. Frequency and topography of cerebral microbleeds in dementia with Lewy bodies compared to Alzheimer's disease. Parkinsonism Relat Disord. 2015;21:1101-1104.

31. Scheid R, Walther K, Guthke T, Preul C, von Cramon DY. Cognitive sequelae of diffuse axonal injury. Arch Neurol. 2006;63:418-424.

32. Bousser M, Tournier-Lasserve E. Cerebral autosomal dominant arteriopathy with subcortical infarcts and leukoencephalopathy: from stroke to vessel wall physiology. $J$ Neurol Neurosurg Psychiatry. 2001;70:285-287.

33. Stojanov D, Vojinovic S, Aracki-Trenkic A, Tasic A, Benedeto-Stojanov D, Ljubisavljevic $S$, et al. Imaging characteristics of cerebral autosomal dominant arteriopathy with subcortical infarcts and leucoencephalopathy (CADASIL). Bosn J Basic Med Sci. 2015;15:1-8.

34. Lesnik Oberstein SA, van den Boom R, van Buchem MA, van Houwelingen HC, Bakker E, Vollebregt E, et al. Cerebral microbleeds in CADASIL. Neurology. 2001;57:1066-1070.

35. Dichgans M, Holtmannspötter M, Herzog J, Peters N, Bergmann M, Yousry TA. Cerebral microbleeds in CADASIL: a gradient-echo magnetic resonance imaging and autopsy study. Stroke. 2002;33:67-71.

36. Lee JS, Ko K, Oh JH, Park JH, Lee HK, Floriolli D, et al. Cerebral microbleeds, hypertension, and intracerebral hemorrhage in cerebral autosomal-dominant arteriopathy with subcortical infarcts and leukoencephalopathy. Front Neurol. 2017;8:203.

37. Nannucci S, Rinnoci V, Pracucci G, MacKinnon AD, Pescini F, Adib-Samii P, et al. Location, number and factors associated with cerebral microbleeds in an Italian-British cohort of CADASIL patients. PLoS One. 2018;13:eo190878.

38. Mehat A, Hughes DA. GeneReviews ${ }^{\circledR}$. University of Washington [serial online] 2002 Aug [cited 2017 Jan 5]. Available from http:// www.ncbi.nlm.nih.gov/books/NBK1292/.

39. Reisin RC, Romero C, Marchesoni C, Nápoli G, Kisinovsky I, Cáceres G, et al. Brain MRI findings in patients with Fabry disease. J Neurol Sci. 2011;305:41-44.

40. Kono Y, Wakabayashi T, Kobayashi M, Ohashi T, Eto Y, Ida $\mathrm{H}$, et al. Characteristics of cerebral microbleeds in patients with Fabry disease. J Stroke Cerebrovasc Dis. 2016;25:1320-1325.

41. Gould DB, Phalan FC, van Mil SE, Sundberg JP, Vahedi $\mathrm{K}$, Massin $\mathrm{P}$, et al. Role of COL4A1 in small-vessel disease and hemorrhagic stroke. N Engl J Med. 2006;354:1489-1496.

42. Weng YC, Sonni A, Labelle-Dumais C, de Leau M, Kauffman WB, Jeanne M, et al. COL4A1 mutations in patients with sporadic late-onset intracerebral hemorrhage. Ann Neurol. 2012;71:470-477. 
43. Lanfranconi S, Markus HS. COL4A1 mutations as a monogenic cause of cerebral small vessel disease: a systematic review. Stroke. 2010;41:e513-e518.

44. Fischer M, Schmutzhard E. Posterior reversible encephalopathy syndrome. J Neurol. 2017;264:1608-1616.

45. Mechtouff L, Piegay F, Traclet J. Tacrolimus-related cerebral microbleeds after lung transplantation. Case Rep Transplant. 2013;2013:708961.

46. Yu WL, Leung T, Soo Y, Lee J, Wong KS. Thrombotic thrombocytopenic purpura with concomitant small- and large-vessel thrombosis, atypical posterior reversible encephalopathy syndrome and cerebral microbleeds. Oxf Med Case Reports. 2015;2015:179-182.

47. McKinney AM, Sarikaya B, Gustafson C, Truwit CL. Detection of microhemorrhage in posterior reversible encephalopathy syndrome using susceptibility-weighted imaging. AJNR Am J Neuroradiol. 2012;33:896-903.

48. Baddour LM, Wilson WR, Bayer AS, Fowler VG Jr, Tleyjeh IM, Rybak MJ, et al. Infective endocarditis in adults: diagnosis, antimicrobial therapy, and management of complications: a scientific statement for healthcare professionals from the American Heart Association. Circulation. 2015;132:1435-1486.

49. Klein I, Iung B, Labreuche J, Hess A, Wolff M, MessikaZeitoun D, et al. Cerebral microbleeds are frequent in infective endocarditis: a case-control study. Stroke. 2009;40:34613465 .

50. Okazaki S, Sakaguchi M, Hyun B, Nagano K, Tagaya M, Sakata Y, et al. Cerebral microbleeds predict impending intracranial hemorrhage in infective endocarditis. Cerebrovasc Dis. 2011;32:483-488.

51. Hess A, Klein I, Iung B, Lavallée P, Ilic-Habensus E, Dornic $\mathrm{Q}$, et al. Brain MRI findings in neurologically asymptomatic patients with infective endocarditis. AJNR Am J Neuroradiol. 2013;34:1579-1584.

52. Iung B, Tubiana S, Klein I, Messika-Zeitoun D, Brochet E, Lepage L, et al. Determinants of cerebral lesions in endocarditis on systematic cerebral magnetic resonance imaging: a prospective study. Stroke. 2013;44:3056-3062.

53. Kim JS. Moyamoya disease: epidemiology, clinical features, and diagnosis. J Stroke. 2016;18:2-11.

54. Kikuta K, Takagi Y, Nozaki K, Sawamoto N, Fukuyama H, Hashimoto N. The presence of multiple microbleeds as a predictor of subsequent cerebral hemorrhage in patients with moyamoya disease. Neurosurgery. 2008;62:104-111.

55. Sun W, Yuan C, Liu W, Li Y, Huang Z, Zhu W, et al. Asymp- tomatic cerebral microbleeds in adult patients with moyamoya disease: a prospective cohort study with 2 years of follow-up. Cerebrovasc Dis. 2013;35:469-475.

56. Wenz H, Wenz R, Maros M, Ehrlich G, Al-Zghloul M, Groden C, et al. Incidence, locations, and longitudinal course of cerebral microbleeds in European moyamoya. Stroke. 2017;48:307-313.

57. Rabin BM, Meyer JR, Berlin JW, Marymount MH, Palka PS, Russell EJ. Radiation-induced changes in the central nervous system and head and neck. Radiographics. 1996;16:10551072.

58. Tanino T, Kanasaki Y, Tahara T, Michimoto K, Kodani $\mathrm{K}$, Kakite S, et al. Radiation-induced microbleeds after cranial irradiation: evaluation by phase-sensitive magnetic resonance imaging with 3.0 tesla. Yonago Acta Med. 2013;56:712.

59. Lupo JM, Molinaro AM, Essock-Burns E, Butowski $\mathrm{N}$, Chang SM, Cha S, et al. The effects of anti-angiogenic therapy on the formation of radiation-induced microbleeds in normal brain tissue of patients with glioma. Neuro Oncol. 2016;18:87-95.

6o. Shen Q, Lin F, Rong X, Yang W, Li Y, Cai Z, et al. Temporal cerebral microbleeds are associated with radiation necrosis and cognitive dysfunction in patients treated for nasopharyngeal carcinoma. Int J Radiat Oncol Biol Phys. 2016;94:1113-1120.

61. Roddy E, Sear K, Felton E, Tamrazi B, Gauvain K, Torkildson J, et al. Presence of cerebral microbleeds is associated with worse executive function in pediatric brain tumor survivors. Neuro Oncol. 2016;18:1548-1558.

62. Armstrong FD. Evidence of cerebral microbleeds and neurocognitive impairment following cranial radiation therapy for pediatric brain tumors: a new opportunity for improved care. Neuro Oncol. 2016;18:1470-1471.

63. Song TJ, Park JH, Choi KH, Chang Y, Moon J, Kim JH, et al. Moderate-to-severe obstructive sleep apnea is associated with cerebral small vessel disease. Sleep Med. 2017;30:36-42.

64. Koo DL, Kim JY, Lim JS, Kwon HM, Nam H. Cerebral microbleeds on MRI in patients with obstructive sleep apnea. J Clin Sleep Med. 2017;13:65-72.

65. Treadwell SD, Robinson TG. Cocaine use and stroke. Postgrad Med J. 2007;83:389-394.

66. Shoamanesh A, Catanese L, Romero JR, Lau H, Babikian VL, Benavente OR, et al. High prevalence of cerebral microbleeds in inner city young stroke patients. J Stroke Cerebrovasc Dis. 2016;25:733-738. 
67. Rutgers DR, Saliou G, Ducreux D. Microbleeds in thrombotic thrombocytopenic purpura. J Neuroradiol. 2008;35:187188.

68. Noorbakhsh-Sabet N, Zand R. Thrombotic thrombocytopenic purpura with concomitant progressive cerebral microbleeds. J Stroke Cerebrovasc Dis. 2016;25:e214-e215.

69. Wijdicks EF, Silbert PL, Jack CR, Parisi JE. Subcortical hemorrhage in disseminated intravascular coagulation associated with sepsis. AJNR Am J Neuroradiol. 1994;15:763-765.

70. Neligan A, Rajakulendran S, Nortley R, Manji H. Extensive cerebral microhemorrhages caused by acute disseminated intravascular coagulation secondary to sepsis. JAMA Neurol. 2014;71:510-511.

71. Trevino-Peinado C, Zubieta JL, Fernández MM. Subcortical microbleeds in disseminated intravascular coagulation mimicking amyloid angiopathy. J Neuroimaging. 2015;25:660-661.

72. DeBakey ME, Henly WS, Cooley DA, Morris GC Jr, Crawford ES, Beall AC Jr. Surgical management of dissecting aneurysms of the aorta. J Thorac Cardiovasc Surg. 1965;49:130149.

73. Nakamura S, Misumi I, Koide S. Cholesterol embolism as a complication of aortic dissection. Circulation. 1999;100:e48-e50.

74. Braem L, Paule P, Héno P, Morand JJ, Mafart B, La Folie T, et al. Thoracic aortic dissection revealed by systemic cholesterol embolism. Ann Cardiol Angeiol (Paris). 2006;55:300303.

75. Müller C, Rahn BA, Pfister U, Meinig RP. The incidence, pathogenesis, diagnosis, and treatment of fat embolism. Orthop Rev. 1994;23:107-117.

76. Kamenar E, Burger PC. Cerebral fat embolism: a neuropathological study of a microembolic state. Stroke. 1980;11:477-484.

77. Lee J. Gradient-echo MRI in defining the severity of cerebral fat embolism. J Clin Neurol. 2008;4:164-166.

78. Mossa-Basha M, Izbudak I, Gurda GT, Aygun N. Cerebral fat embolism syndrome in sickle cell anaemia/ $\beta$-thalassemia: importance of susceptibility-weighted MRI. Clin Radiol. 2012;67:1023-1026.

79. Haller S, Vernooij MW, Kuijer JPA, Larsson EM, Jäger HR, Barkhof F. Cerebral microbleeds: imaging and clinical significance. Radiology. 2018;287:11-28.

8o. Renard D. Cerebral microbleeds: a magnetic resonance imaging review of common and less common causes. Eur J Neurol. 2018;25:441-450. 